\title{
BUILDING A MODEL FOR RESERVOIR CHARACTERISATION IN GIS USING MACHINE LEARNING
}

\author{
Ayush Singh $^{1 *}$, Priyanshu Bhardwaj ${ }^{1}$, Susham Biswas ${ }^{2}$ \\ ${ }^{1}$ Department of Petroleum Technology, Rajiv Gandhi Institute of Petroleum Technology, (epe18008, epe16024, epe16043) \\ @rgipt.ac.in \\ ${ }^{2}$ Faculty Geoinformatics, Rajiv Gandhi Institute of Petroleum Technology(susham@rgipt.ac.in)
}

KEYWORDS: Reservoir, Interpolation, 3D Model. GIS, MATLAB

\begin{abstract}
:
The authors have tried to build a 3D model for reservoir characterization. The model is planned in such a way to accommodate multiple wells with their Petro-physical data spatially using different grids and then integrating the data to determine the reservoir characteristics for unknown locations in 3D. Initially, the model is planned using well log data of Equinor Volve field (central part of North Sea). Computational analysis for reservoir characterization was conducted in GIS type platform using ML approach integrating with MATLAB and PYTHON plugins. The model provides an opportunity to determine reservoir characteristics at desired $\mathrm{X}, \mathrm{Y}, \mathrm{Z}$ coordinate. However, there remain important challenges of deciding the size of the 3D grid, vis a vis availability of data, assigning the data to grid cell, assigning weights to each populated grid, and ascertainment of the model to relate a surface between known grid cell, and checking the accuracy of a fit surface from various directions in 3D. On analysis of the grid data for wells, it came out that for few places the values are more homogeneous while at other, they are abruptly changing. Various methods of reservoir characterization have been referred to which use a different technique of data evaluation at unknown points. Once the grids were populated with known data, unknown grid locations were ascertained with interpolation such as nearest neighbour and linear method. Initially, interpolation was tried to be made in X-Y, X-Z, Y-Z plane and then at a plane in any direction in 3D. Multi interpolations have been used in the model that enables authors to view a desired surface in the reservoir to suggest the best possible direction of drilling to hit the correct pay zones. Even though uncertainty will be encountered but authors have strived to suggest a probable way to proceed from the available data.
\end{abstract}

\section{INTRODUCTION}

Oil and gas operations aim towards minimising the overall expenditure and maximising its total profit. Typically, the journey of a well starts from collection of data, which is done in the exploration stage, in the form of seismic surveys, petrophysical well logs, geographical analysis and lithology. The exploration team decides whether to embark ahead or abort the field. What is the key here? You guessed it right, 'DATA'. How delicately the collected data is analysed plays a key role in the future of a well. Drilling a single well requires billions to be invested, one cannot just aim anywhere in the geological field and hope to hit the best pay zones. An Oil/Gas reservoir, which is the result of billions of years of decay and decomposition of dead organic matter, is what that needs to be characterised with the help of data.

Generally, a reservoir is defined by the amount of fluid it can hold in the effective pores, known as porosity and whether or not the pores are interconnected for effective flow, known as permeability. Water saturation, the compressibility of pores and matrix, and resistivity also help in the description of a reservoir. Recovery from the reservoir depends on how well these petrophysical quantities are in your favour, but whether or not you are utilising this knowledge to strike the right spot remains the key. Suppose a unit of a reservoir contains all the information in it, it is a thumb rule that the nearest unit will have a certain effect of that unit.

If the data is used to interpolate/extrapolate the field then we obtain certain information about new locations. It is bound to increase efficiency in the next drilling operation. Starting from the exploratory well and going towards production every ounce of new data will enhance the next spot selection. Owing to the limitation of data it becomes important how we integrate welllog, geological, seismic and engineering data (Ma, Zee,2011). This paper is an attempt for using this concept and thus develop a model that does so. Errors encountered due to data impurity is a challenge to handle. Data must be well prepared for fitting the model. Regression approach, just like interpolation, is one of the statistical approaches being used for reservoir characterisation. Combined use of clustering, regression and component analysis was found in literature. (Gudmundsdottir and Horne,2018). Prediction using kriging, a form of regression, is also being used (Vasquez,2014). For limited data often interpolation using inverse distance (nearest neighbour) gives better result than kriging. (Otchere,2016), which is the case with considered data. Reservoir simulations software are $\mathrm{n}$ in number and they offer various features for visualising it, but our approach attempts to set a new way ahead. The authors want to work to provide solutions for future energy.

\section{LITERATURE REVIEW.}

2.1 Field report (Norwegian petroleum Directorate,2015)

\subsubsection{Developments}

Volve is a field in the central part of the North Sea, five kilometres north of the Sleipner Øst field. The water depth is 80 metres. Volve was discovered in 1993, and the plan for development and operation was approved in 2005. The field was developed with a jack-up processing and drilling facility. The vessel "Navion Saga" was used for storing stabilised oil. Production from the reservoir started in 2008.

\footnotetext{
* Corresponding author
} 


\subsubsection{Reservoir}

Volve produced oil from the sandstone of Middle Jurassic age in the Hugin Formation. The reservoir is at a depth of 2,7003,100 metres. The western part of the structure is heavily faulted and communication across the faults is uncertain.

\subsubsection{Performance}

When the Volve field started well drilling in May 2007 and came into production the following year, life expectancy was from 3-5 years. New wells were being drilled up until 2012-13, which contributed to the increased recovery rate and extended life of the field. The field has been functioning for over eight years and brings in about 9.5 million barrels of oil than expected for development and operational programs. Overall, Volve achieved a return rate of $54 \%$.

\subsubsection{Recovery and Transport}

The field was produced with water injection for pressure support. The oil was exported by tankers and the gas was transported to the Sleipner A facility for further export.

\subsubsection{Status}

Volve was shut down in 2016 by a decision of the partners: Statoil (now Equinor), ExxonMobil and Bayern Gas (Now Spring Energy). The facility was removed in 2018.

$\begin{array}{lrrr} & \text { DEPTH } & \text { PHIF } & \text { KLOGH } \\ \text { count } & 12301.000000 & 12301.000000 & 12301.000000 \\ \text { mean } & 3772.255923 & 0.118287 & 1042.338642 \\ \text { std } & 421.447942 & 0.089431 & 12385.494276 \\ \text { min } & 3102.102000 & 0.000000 & 0.000000 \\ 25 \% & 3419.100000 & 0.037500 & 0.001000 \\ 50 \% & 3665.067600 & 0.102709 & 0.033000 \\ 75 \% & 4133.697600 & 0.191609 & 2.985000 \\ \text { max } & 4618.177200 & 0.352600 & 760405.250000\end{array}$

Table 1. The statistical description of porosity (PHIF) and permeability (KLOGH). Number of points, mean, standard deviation, minimum and quartile values are shown. (Modified from the dataset. (Equinor, 2018))

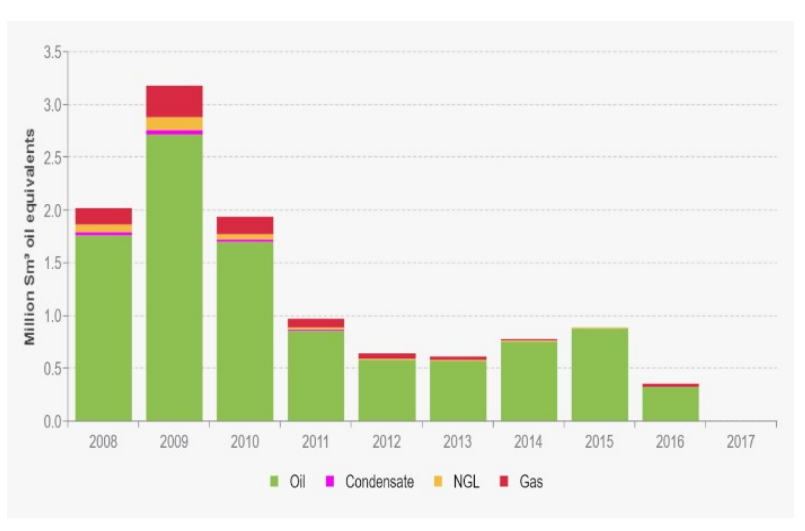

Figure 1. Pictorial representation of production data from the field (Norwegian petroleum directorate,2015). The X-axis shows the years and the y-axis shows the equivalents of hydrocarbon produced in millions standard cubic meter.
Location: About 200 kilometres west of Stavanger at the southern end of the Norwegian sector.

Production start: 12 February 2008.

Production end: 17 September 2016.

Production: Oil.

\subsection{Data report (Equinor,2018)}

The dataset comprises approximately 40,000 files from the Volve field. The data has been released to provide a scientific opportunity for students and scientists. Geophysical interpretation, Production data, Reservoir model through ECLIPSE and RMS, Seismic data, Well Logs, Well Technical data and Well Real-time drilling data are the information provided.

Well logs serve the purpose of the research work, they contain a variety of logs: mud logs, petrophysical logs, core, biostratigraphy, geochemical and corresponding reports from 3 exploration wells and 21 production/injection wells.

Out of this, the crux is in the petrophysical log. It consists of ". Las" format files describing the parameters. Depth wise data of $\operatorname{logs}$ is given such as porosity, horizontal permeability, saturation, the volume of shale, etc. in each folder. Units, not defined values and other vital information is given in the files. Not all folders contained all parameters and this requires the primary task of data management and few assumed methods for conversion to 3 -d format.

Well 15/9 F-1

\begin{tabular}{|l|l|l|}
\hline DEPTH & PHIF & KLOGH \\
\hline 3319.7 & 0.0200 & 0.0010 \\
\hline 3319.8 & 0.0200 & 0.0010 \\
\hline 3319.9 & 0.0200 & 0.0017 \\
\hline 3320.0 & 0.0238 & 0.0306 \\
\hline 3320.1 & 0.0413 & 0.1252 \\
\hline
\end{tabular}

Well 15/9 F-12

\begin{tabular}{|l|l|l|}
\hline DEPTH & PHIF & KLOGH \\
\hline 3102.1020 & 0.2699 & 0.001 \\
\hline 3102.2544 & 0.2680 & 0.001 \\
\hline 3102.4062 & 0.2662 & 0.001 \\
\hline 3102.5592 & 0.2651 & 0.001 \\
\hline 3102.7116 & 0.2651 & 0.001 \\
\hline
\end{tabular}

Well 15/9 F-SR

\begin{tabular}{|l|l|l|}
\hline DEPTH & PHIF & KLOGH \\
\hline 3568.9032 & 0.185253 & 6.780 \\
\hline 3569.0556 & 0.183710 & 5.932 \\
\hline 3569.2080 & 0.174967 & 2.781 \\
\hline 3569.3604 & 0.167252 & 1.426 \\
\hline 3569.5128 & 0.150795 & 0.343 \\
\hline
\end{tabular}

Table 2. Represents values of test wells shown in figure 2. first five points representing porosity and permeability out of 12,301 total points in all three wells (modified).

\section{METHODOLOGY}

\subsection{Data preparation}

As analysed in the data report, porosity was used to study the devised model. Porosity is an important petrophysical parameter to characterise a reservoir. Its data values were most consistent throughout the dataset. Pertaining to the fluctuations, NAN 
values and errors in the porosity data were cleaned. NAN values in different logs were -999.25 . Data of three wells, 15/9 F-1, 15/9 S\&SR and 15/9 F-12, were taken and grouped. Total points after cleaning the data were 12,301 .

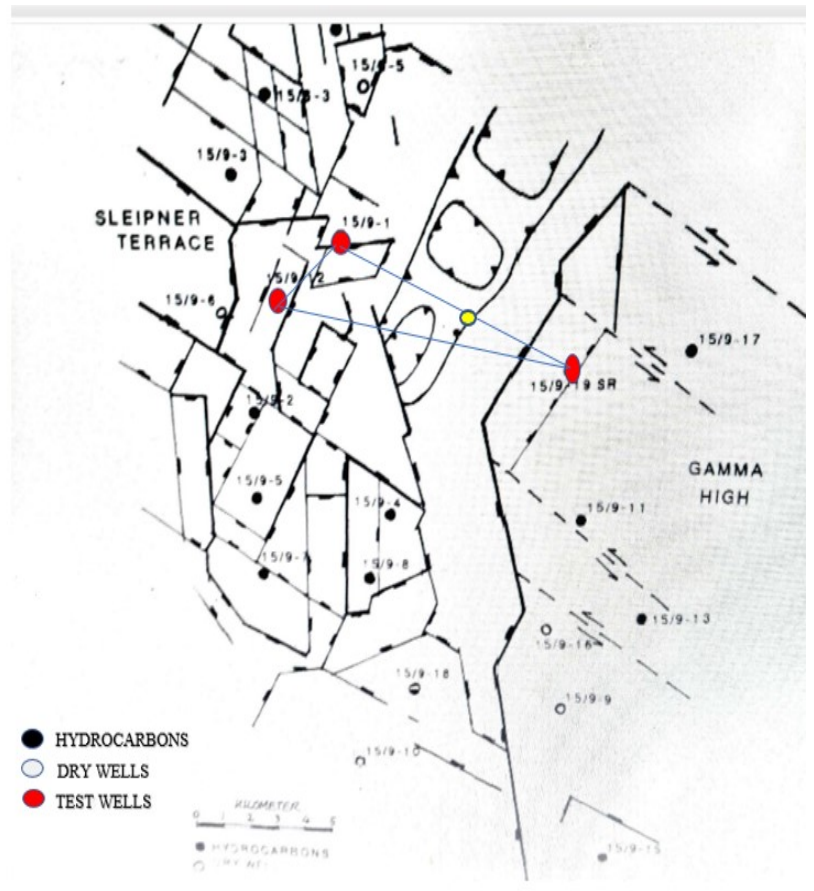

Figure 2. Map displaying the location of various wells. black circle represent hydrocarbon well and the white one represents dry wells. Red circle are the test wells that have been used for testing the model. yellow circle depicts the origin. On scale unit, $3 \mathrm{~cm}$. is equal to $5 \mathrm{~km}$. (Figure was modified. (Equinor,2018))

Well 15/9 F-1, Well 15/9 F-12, Well 15/9 F-SR

Assumed origin (every coordinate is with respect to it)

\subsection{Interpolation Method and Slicing Techniques}

The prepared data is fitted on the model. The model involves multiple interpolations. Initially, the data is fed as discrete and scattered(discrete) form to a scattered interpolant object. The depth serves as $\mathrm{z}$ coordinates while $\mathrm{x}$ and $\mathrm{y}$ coordinates were traced from the figure (5.). The result is obtained for a meshgrid that is the first stepping stone for the characterisation. The coordinates of mesh-grid(a) were:

$\mathbf{X}:(-5.5,4.5)$ with step of 0.5 unit

$\mathbf{Y}:(-2,2)$ with step of 0.5 -unit

$\mathbf{Z}:(3000,3300)$ with a step of 1 unit

$1 \mathrm{unit}=1 \mathrm{~km}$.

The interpolation was carried out using the nearest neighbour method. This suits to a reservoir unit due to the effect of one grid unit on another. By this approach, we can pick any axis parallel to $\mathrm{x}, \mathrm{y}$ or $\mathrm{z}$.

The author aims to interpolate in any direction possible within the mesh-grid. For this, the interpolated mesh-grid, which was generated from scattered interpolation, was fitted on a 3- D interpolant object as a gridded data. A finer mesh grid(b) was generated with coordinates:

$\mathbf{X}:(-2.5,2.5)$ with step of 0.25 units

Y: $(-1.5,1.5)$ with step of 0.25 units

Z: $(3100: 3250)$ with step of 0.5 units $\quad 1$ unit $=1 \mathrm{~km}$.

This interpolation can be carried out with various techniques like spline, cubic, nearest and maxima. The difference lies in the working of the algorithm.
The mesh-grids were fitted on the slice object with different (X, Y, Z) slices. This enables the selection of a single plane or multiple planes to view a petrophysical parameter. A surface that lies within the interval of mesh-grids can be viewed after its characterisation, by fitting into this model.

\subsection{Validation}

The results that were obtained using the model were crossvalidated. Out of the three test wells the well 15/9 F-12 was omitted and a new data was generated using the other two test wells and the mesh-grid(a). This new data was fitted on the model to obtain results at the same location as that of the well 15/9 F-12.

Mean square error was calculated between the original data and the interpolated one, to show the accuracy of the model.

\section{COMPUTATION}

\subsection{Conversion of Data to 3-d}

The $(\mathrm{x}, \mathrm{y})$ coordinates of the test wells are obtained by assuming origin at the centre of the line joining the well SR and F-1 of $15 / 9$, as shown in figure 4 . Thereby the coordinates of test wells were obtained from the figure (4.) as follows:

15/9 S\&SR: $(4.5,-2)$

15/9 F-1: $(-4.5,2)$

15/9 F-12: $(-5.3,0.8)$

While working with the validation part, the interpolated point was located at $(-1,0)$. porosity values.

NAN values were replaced with mean. But this strategy was not very fruitful so the rows containing the NAN values were eliminated. The total points after data manipulation were 6782 , 2873 and 2648 in SR, F-1, F-12 wells respectively.

\subsection{Algorithms}

\subsubsection{Scattered Interpolation}

It was used to perform interpolation on the set of scattered 3-D data. After object creation, it can be used to evaluate interpolated values at different query points. Scattered interpolant uses a Delaunay triangulation (Ahmed,2010, p.281) of the data, so can be sensitive to scaling issues in the sample points. When this occurs one can use normalisation to rescale the data and improve the results. A $2 \mathrm{D}$ dataset is triangulated and a 3-D dataset is tetrahedrised (Amidror, April 2002), thus the interpolation scheme is attached to the tetrahedron/triangle. These methods are always local. MATLAB was used to perform all operations (MATLAB,2019).

$\mathrm{F}=$ scatteredInerpolant $(\mathrm{x}, \mathrm{y}, \mathrm{z}, \mathrm{v}$, function $)$

Where $(\mathrm{x}, \mathrm{y}, \mathrm{z})$ are coordinates, $\mathrm{v}$ is the value, and the function signifies the approach.

\subsubsection{3-D Gridded Interpolation}

It is used to perform interpolation on a 3-D gridded data in a mesh-grid format. For interp3, a full grid consists of three arrays whose elements represent a grid of points that define a region in $R^{3}$. The first array contains the $x$-coordinates, the second array contains the $y$-coordinates, and the third array contains the $z$-coordinates.

$\mathrm{F}=$ interp3(X, Y, Z, V, P, Q, R)

Where $(\mathrm{x}, \mathrm{y}, \mathrm{z})$ are coordinates, $\mathrm{v}$ is the value, $(\mathrm{P}, \mathrm{Q}, \mathrm{R})$ are query points, and the function signifies the approach. 


\subsubsection{Functions}

Nearest Neighbour- applied for generation of mesh grid(a). Linear- applied for generation of mesh grid(b).

\section{RESULTS}

The authors computed the interpolated porosity at the point $(-1,0)$ using the model. Figure 3. shows the result.

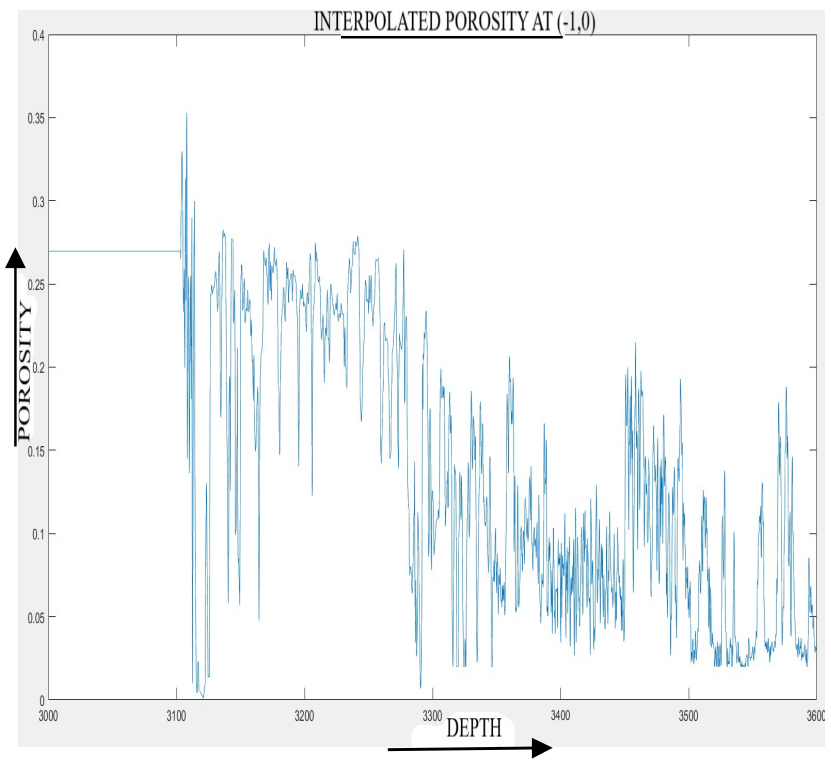

Figure 3. Interpolated porosity(y-axis) at $(-1,0)$ concerning the depth in km. (x-axis)

Validation result obtained at well 15/9 F-12 is shown in Figure 4. Mean square error between the true porosity value and interpolated porosity value of well $15 / 9 \mathrm{~F}-12$ was 0.03287165 .

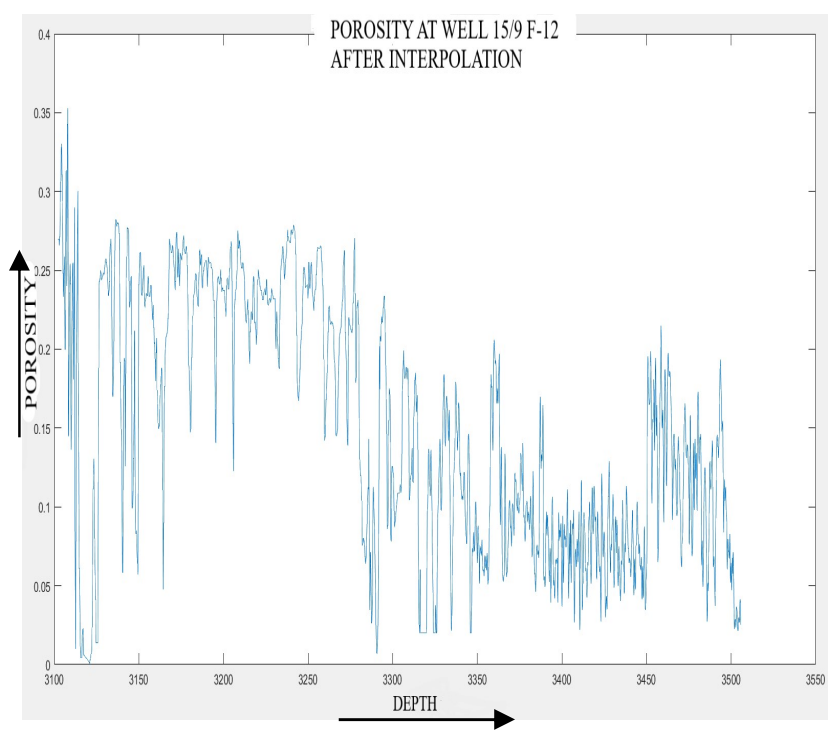

Figure 4. Interpolated porosity(y-axis) at well 15/9 F-12 concerning the depth in $\mathrm{km}(\mathrm{x}$-axis).

Figure 5. was obtained using scattered interpolant object with the nearest neighbour method. Next, the authors fitted the generated results to the 3-D interpolation object and thus gained access to all the planes possible within the mesh-grid. Colour bar shows a range of porosity.
Figure 5. has the following specifications.

X: $(-5.5,4.5)$, step of $0.5 \mathrm{~km}$.
$Y:(-1.5,1.5)$, step of $0.25 \mathrm{~km}$
$Z:(3100: 3250)$, step of $0.5 \mathrm{~km}$

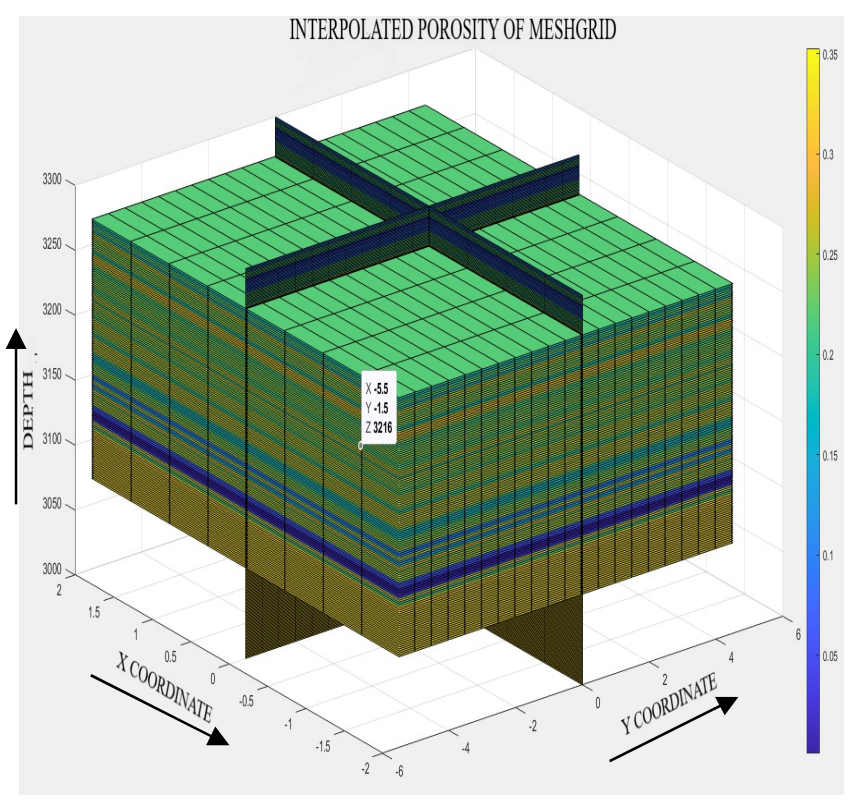

Figure 5. Reservoir porosity (displayed on colour-bar) of interpolated mesh-grid(a) whose coordinates are mentioned above this figure.

Figure 6. and 7. show how a curve is visualised in the reservoir. The grid spacing was controlled by altering the spaces or the interval between depths. Owing to computational limitation the curve has covered a small gap in the reservoir. The difference between the two curves is the grid spacing. Figure 7. is more refined.

Figure 6. has the following specifications:

$\mathrm{X}, \mathrm{Y}:(-1.5,1.5)$, step of $0.2 \mathrm{~km}$

$\mathrm{Z}:\left(\left(\mathrm{X} \cdot{ }^{\wedge} 2-\mathrm{y}^{\wedge} 2\right)+3150\right)$.

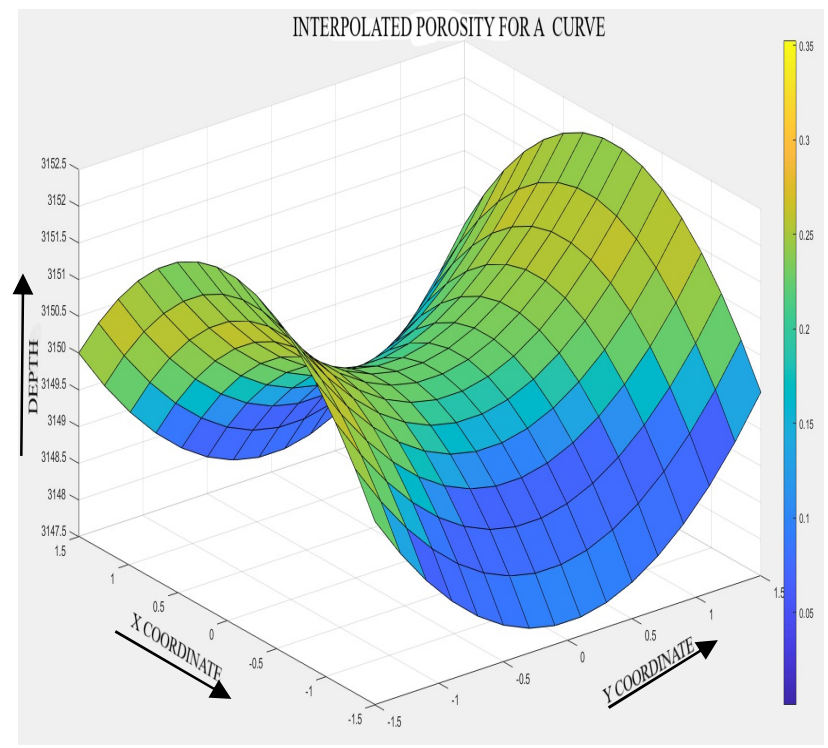

Figure 6. Section of the reservoir using a curved surface showing porosity (displayed on colour-bar). 
Figure 7. and 8. have the following specifications:

$\mathrm{X}, \mathrm{Y}:(-1.5,1.5)$ step of $0.1 \mathrm{~km}$

$\mathrm{Z}:\left(\left(\mathrm{X} \cdot{ }^{\wedge} 2-\mathrm{y}^{\wedge} 2\right)+3150\right)$.

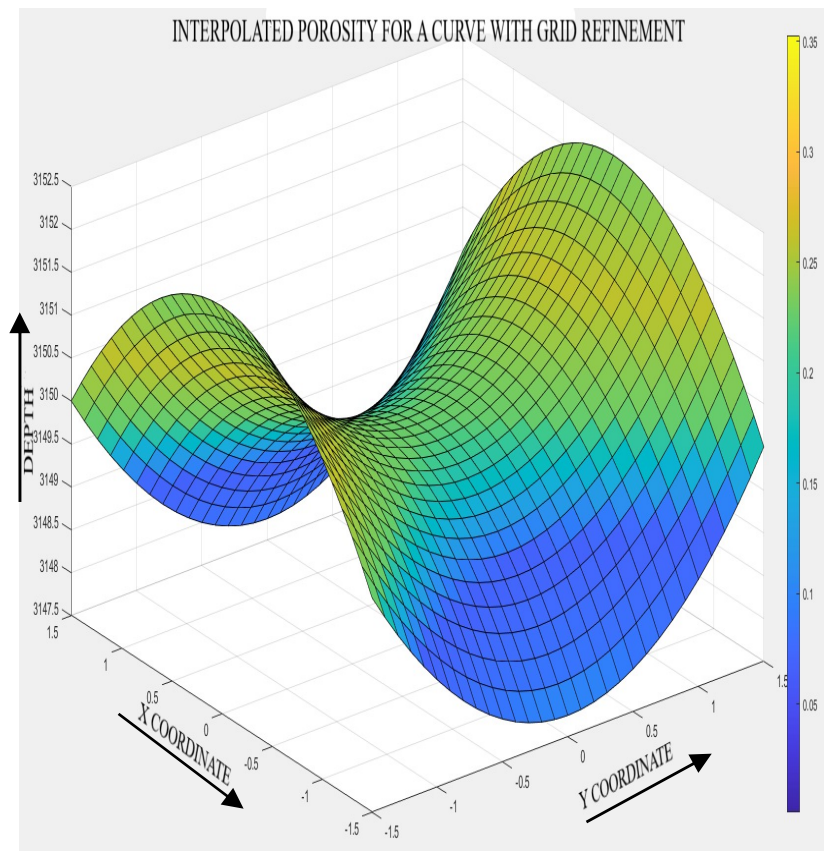

Figure 7. Grid refinement of figure 6.

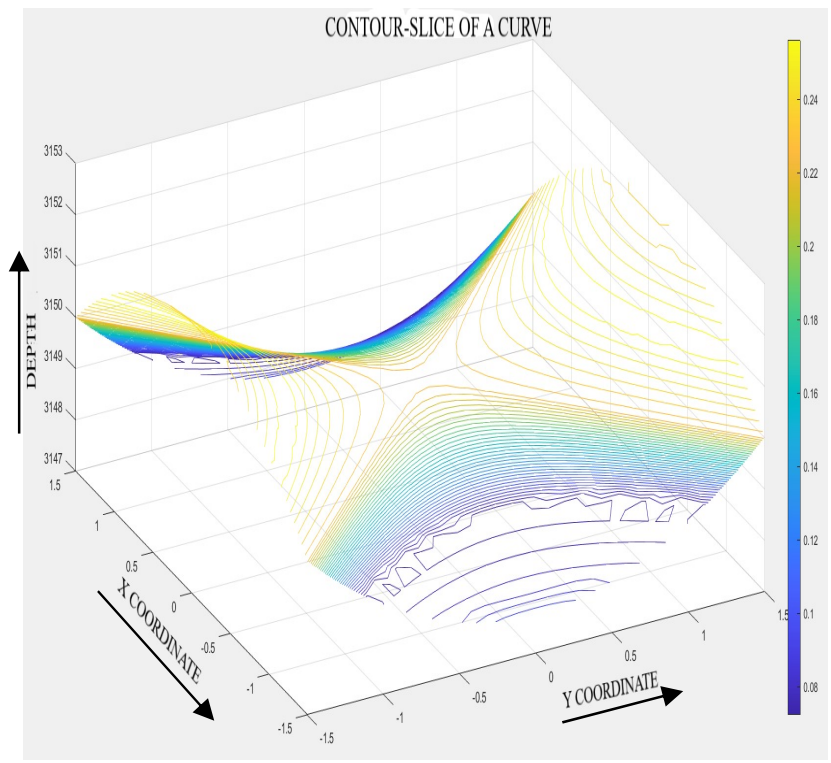

Figure 8. Contour slice of the curved surface(of figure 7.) showing porosity variation (displayed on colour-bar).

Variation in the petrophysical quantities can be visvalised across the reservoir, within the considered dimensions of meshgrid. Figure 6,7,8 depict the variation for a curved surface while figure 9 . displays variation for a plane surface.

Figure 9. has the following specifications:

$\mathrm{X}, \mathrm{Y}:(-1.5,1.5)$, step of $0.1 \mathrm{~km}$

$Z:((X+Y)+3200)$

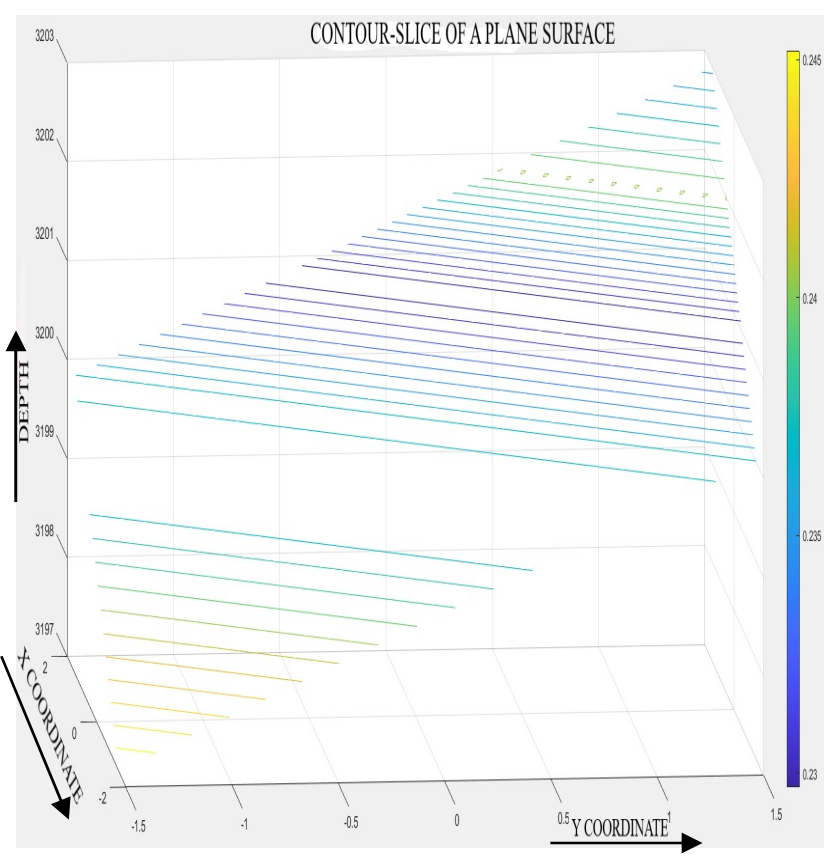

Figure 9. Contour-slice of a plane surface showing porosity variation (displayed on colour-bar).

\section{CONCLUSION}

The authors have successfully used interpolation methods in the field of reservoir study by attempting to characterize the reservoir, and determine the petrophysical parameters for unknown locations below the ground using a novel technique. Few key findings are given below:

Data impurity can hamper the performance of the model greatly. Interpolation methods especially used in this paper, involving triangulation, are highly vulnerable to the presence of outliers. So, the dataset must be normalised and scaled. It was even interesting to know how spatial data analysis is quite different. Normalisation impacts the petrophysical parameters, but the depth for these values remain the same, by adding a directional bias. Thus, giving important results to analyse after fitting. Excluding such outliers was a better approach.

Various approaches are available to deal prediction of parameters at unknown point using scattered data. But this situation demanded the use of nearest neighbour because of the behaviour of a petrographic unit. Further linear fitting helped the cause after 3-d data generation. Multiple interpolations proved to be a unique way that the authors found to deal with such cases.

Reservoir characterisation was achieved in all the possible direction within the domains of chosen mesh-grid. Planes parallel to $\mathrm{x}, \mathrm{y}, \mathrm{z}$ or in any direction was obtained using the model.

Further work can be done on developing models by exploring the plethora of data visualisation and prediction tool.

\section{REFERENCES}

Ahmed, T. (2010). Fundamentals of rock properties. In Reservoir Engineering Handbook(4th ed., pp. 274-281). Burlington, USA. https://doi.org/10.1016/C2009-0-30429-.

Amidror, I. (April 2002). Scattered data interpolation methods for electronic imaging systems: a survey. Journal of Electronic Imaging, 157-176. 
Equinor. (2018, October 22). Equinor Data Portal Beta. Retrieved from $</$ data.equinor.com $>$.

Gudmundsdottir H, Horne R. (2018) Reservoir characterization and prediction modeling using statistical techniques. Proceedings 43rd Workshop on Geothermal Reservoir Engineering, Stanford University, Stanford Cal. USA.

Ma, Zee. (2011). Reservoir Characterization: A look back and ways forward. AAPG Bulletin.

MATLAB. (2019). version 9.7.0 (R2019b). Natick, Massachusetts: The MathWorks Inc.

Norwegian Petroleum Directorate. (2015, February 24). Field: VOLVE<https://www.norskpetroleum.no/en/facts/field/volve/>

Vasquez DA (2014) Geological modelling in GIS for petroleum reservoir characterization and engineering: a 3D GIS-assisted geostatistics approach. An M.Sc Thesis submitted to the Graduate School, University of Southern California, p 58.

Otchere, Daniel. (2016). Static Reservoir Modelling Comparing Inverse Distance Weighting to Kriging Interpolation Algorithm in Volumetric Estimation. Case Study: Gullfaks Field. 10.13140/RG.2.2.23877.35047. 